\title{
Postoperative Ileus
}

\author{
Cristina R. Harnsberger, MD ${ }^{1}$ Justin A. Maykel, MD ${ }^{1}$ Karim Alavi, MD, MPH \\ ${ }^{1}$ Division of Colon and Rectal Surgery, University of Massachusetts, \\ Boston, Massachusetts \\ Address for correspondence Karim Alavi, MD, MPH, Division of Colon \\ and Rectal Surgery, University of Massachusetts, Boston, MA \\ (e-mail: Karim.Alavi@umassmemorial.org).
}

Clin Colon Rectal Surg 2019;32:166-170.

\begin{abstract}
Postoperative ileus (POI) is a common complication following colon and rectal surgery, with reported incidence ranging from 10 to $30 \%$. It can lead to increased morbidity, cost, and length of stay. Although definitions vary considerably in the literature, in its pathologic form, it can be characterized by a temporary inhibition of gastrointestinal motility after surgical intervention due to nonmechanical causes that prevents sufficient oral intake. Various risk factors for development of POI have been identified including increasing age, American Society of Anesthesiologists scores 3 to 4, open approach, operative difficulty, operative duration more than 3 hours, bowel handling, drop in hematocrit or need for a transfusion, increasing crystalloid administration, and delayed mobilization. While treatment is expectant and supportive, significant investigations into strategies to mitigate development of $\mathrm{POI}$ or shorten its duration have been undertaken with mixed results. There is significant evidence to suggest that a

\section{Keywords}

- postoperative ileus

- paralytic ileus

- colorectal complication minimally invasive approach and multimodal pain regimens reduce the development of POI. The beneficial effect of chewing gum, alvimopan, and enhanced recovery after surgery protocols may decrease development of $\mathrm{POI}$ in selected groups of patients who undergo elective colorectal surgery, and shorten time to return of bowel function, but overall, the data remain inconclusive.
\end{abstract}

Postoperative ileus (POI) is a frequent complication after colon and rectal surgery, leading to increased morbidity, cost, and hospital length of stay. Although there is a large variation in reported incidence of POI, which may in part be attributed to a lack of standardized definition, a systematic review and meta-analysis revealed an incidence of 10.3 and $10.2 \%$ for non-randomized controlled trials (RCTs) and RCTs, respectively. ${ }^{1}$ Others have reported its occurrence in 10 to $30 \%$ of patients following abdominal surgery. ${ }^{2}$

Despite its common occurrence, POI remains poorly defined. As such, an international group of colorectal surgeons reached a consensus via a Delphi process that POI can be defined as a temporary inhibition of gastrointestinal motility after surgical intervention due to nonmechanical causes that prevents sufficient oral intake. ${ }^{3}$ The reported duration ranges from 2 to 6 days. $^{2}$ The terminology can be confusing, as some component of POI following abdominal surgery is physiologic. This has led to the use of terms, such as "abnormal POI," “prolonged POI," or "paralytic ileus." A retrospective review of 88 patients who underwent abdominal surgery identified the median duration of POI was 5 days, with an interquartile range of 3 to 6 days; as such, the authors concluded that ileus longer than the third quartile, more than 6 days, served as a better clinical definition of prolonged POI than the 3 days that had previously been suggested. ${ }^{4}$ In the information that follows, the term POI will be used to represent the pathologic state as defined earlier by the expert consensus group of colorectal surgeons, rather than that experienced as part of uncomplicated, normal postoperative recovery.

\section{Pathophysiology}

The etiology of POI is thought to be multifactorial, originating from the surgical stress response. Inflammatory cells are activated and autonomic dysfunction occurs with resulting
Issue Theme Complications and Dilemmas in Colorectal Surgery; Guest Editor: Skandan Shanmugan, MD
Copyright (c) 2019 by Thieme Medical Publishers, Inc., 333 Seventh Avenue, New York, NY 10001, USA. Tel: +1(212) 584-4662.
Dol https://doi.org/ 10.1055/s-0038-1677003. ISSN 1531-0043. 
modulation of gastrointestinal hormone activity. Agonism of gut opioid receptors due to administration of narcotic pain medication, electrolyte derangement, and fluid overload further exacerbates the process. ${ }^{5}$ The pathophysiology is well described by Vather et al, with a final common pathway of impaired contractility and motility, and gut wall edema. ${ }^{6}$

\section{Risk Factors}

Several studies have investigated risk factors for development of POI in patients who undergo colorectal surgery. In a retrospective review of 255 elective colorectal resections in 2011, increasing age (odds ratio [OR]: 1.032, 95\% confidence interval [CI $1.004-1.051 ; p=0.026)$ and increasing drop in pre-topostoperative hemoglobin (OR: $1.043,95 \% \mathrm{CI}$ : 1.002-1.085; $p=0.037$ ) were identified as independent predictors of POI. ${ }^{7}$ In a retrospective single-institution study of 513 segmental colon resection, American Society of Anesthesiologists scores 3 to 4 and duration of surgery $>3$ hours were identified as independent predictors of POI. ${ }^{8}$ Investigation of risk factor analysis was performed in a prospective manner on 327 patients by Vather et al, $27 \%$ of which developed POI. They identified numerous independent predictors including operative difficulty, operative bowel handling, red cell transfusion, increased intravenous crystalloid administration, and delayed first mobilization. These factors were then used to create a risk stratification model. ${ }^{9}$ In addition, an open approach has been clearly shown to be a risk factor in multiple studies, which is further described in the next section. Therefore, the older, sicker patients who undergo long and difficult open operations that require transfusions or excess crystalloid administration are at highest risk. This prompts the question, what measures can be taken to mitigate risk of POI development?

\section{Prevention}

\section{Minimally Invasive Approach}

Multiple studies have demonstrated a protective effect of minimally invasive surgery on the development of POI. ${ }^{8-10}$ An early study randomized 60 patients with colorectal tumors to laparoscopic or open resection, time to first flatus and time to first bowel movement occurred earlier in the laparoscopic group (50 vs. 79 hours, $p<0.01$ and 70 vs. 91 hours, $p<0.01$, respectively). ${ }^{10}$ In another randomized trial of patients 75 years of age or older with cTis-T4a colorectal cancer, patients were randomized to open or laparoscopic surgery with 100 in each group. There was a significantly higher rate of POI in the open group (12 vs. $4 \%$ ) as well as complications, estimated blood loss, and length of stay. ${ }^{11}$ In addition to the well-characterized benefits of laparoscopic surgery in general (less pain, shorter hospital length of stay, quicker return to work, etc.), a reduction in POI following colorectal surgery suggests a minimally invasive approach should be offered even to elderly patients who are candidates.

\section{Enhanced Recovery after Surgery}

The traditional surgical practice of fasting until bowel function has evolved. A systematic review of 15 studies of early post- operative feeding in those who underwent elective open colorectal resection demonstrated no significant difference in total complications and was well tolerated in $86 \%$ of patients. $^{12}$ Enhanced recovery after surgery (ERAS) protocols were developed with variable components, generally including preoperative counseling, early mobilization, early feeding, standardized analgesic regimens, and fluid restriction. The effectiveness of ERAS protocols in reducing POI has been debated. A retrospective analysis of 513 colorectal surgery patients at a single institution demonstrated a protective effect of POI development with $>70 \%$ compliance to the ERAS protocol, but with a $\mathrm{CI}$ that reached 1 (OR: 0.7, 95\% CI: 0.6$1, p=0.031)^{8}$ However, an earlier prospective nonrandomized study of 80 patients who underwent elective colorectal resection from 2003 to 2005 failed to demonstrate a difference in time to first flatus, bowel movement, or length of stay in patients who underwent early feeding, mobilization, and fluid restriction. ${ }^{13}$ Part of the inconclusive results likely stem from a lack of standardization of ERAS protocols after colon and rectal surgery. There are now clinical practice guidelines for enhanced recovery after colon and rectal surgery from the American Society of Colon and Rectal Surgeons and the Society of American Gastrointestinal and Endoscopic Surgeons. ${ }^{14}$ The guidelines are based on the best available evidence and are inclusive but do not dictate specific forms of treatment. As such, it is unlikely that ERAS protocols will become standardized across institutions. Based on current literature and a lack of standardization, a direct causal association between ERAS implementation and decreased POI cannot be established.

\section{Pharmacologic Therapy}

Alvimopan is the U.S. Food and Drug Administration (FDA) approved, peripherally acting mu opioid antagonist for use in preventing POI when started preoperatively in selected patients. Those who have no evidence of bowel obstruction, are not on preoperative narcotics, and are advanced to a minimum of clear liquids following surgery are candidates for its use. In the setting of open abdominal surgery, multiple studies have demonstrated a beneficial effect of alvimopan in recovery of bowel function. A systematic review and metaanalysis of nine randomized trials including 4,075 patients who underwent open abdominal surgery demonstrated a significantly shorter time to first bowel movement. ${ }^{15}$ Although there are data supporting its utility in shortening the duration of POI, the logistics involved in procuring, storing, and dispensing the drug in addition to the associated cost for up to 15 doses as approved by the FDA may prevent its routine use. ${ }^{16}$ In a review of 660 elective colon resections, patients on an ERAS pathway demonstrated a faster return of bowel function by 0.6 days and a lower incidence of POI. ${ }^{17}$ The question of whether alvimopan has any additional value in patients who undergo laparoscopic colorectal surgery with an advanced recovery pathway has also been addressed. Keller et al performed a case-matched analysis of patients who underwent laparoscopic colorectal resection with enhanced recovery in 321 patients who received alvimopan and 321 control patients. They found no difference in ileus or length of stay. ${ }^{18}$ Alvimopan appears to contribute to a 
reduction in POI most notably in patients who undergo open abdominal surgery, while there is debate over its effectiveness in patients who undergo laparoscopic colorectal surgery and adhere to ERAS protocols.

Gastrografin was postulated to have an advantageous effect on reducing bowel edema and thereby shortening the duration of POI, as it is a hyperosmolar, orally administered, contrast agent. This theory was tested in a double-blinded RCT with 80 patients in each arm; patients in the intervention group were given $100 \mathrm{~mL}$ of Gastrografin on postoperative day 1 . While Gastrografin did appear to accelerate time to flatus or stool ( 18.9 vs. 32.7 hours; $p=0.047$ ), it did not significantly affect time to resolution of nausea and vomiting, consumption of oral diet, nasogastric output, analgesia, antiemetic use, complications, or length of stay. The authors concluded that it was not clinically useful in shortening POI characterized by upper and lower gastrointestinal symptoms. ${ }^{19}$

Multimodal analgesic regimens when used in combination with early enteral feeding have demonstrated benefit in restoration of gastrointestinal motility. A retrospective analysis of 88 patients who underwent abdominal surgery identified increasing total opiate dose as a risk factor for development of POI. ${ }^{4}$ A prospective, randomized, doubleblinded trial of 102 patients who underwent elective colorectal resection received either a morphine patient-controlled analgesia (PCA) or morphine PCA with the addition of ketorolac. Those who used ketorolac had $18.3 \%$ less morphine in 72 hours, significantly shorter time to passage of flatus or first bowel movement, and 5.25 times decreased risk of POI. ${ }^{20} \mathrm{~A}$ prospective, double-blinded, controlled study was conducted comparing morphine PCA with placebo versus morphine PCA with valdecoxib (COX-2 inhibitor) given pre- and postoperatively every 24 hours in patients who underwent elective colorectal resection. ${ }^{21}$ Their results demonstrated shorter time to first bowel movement (72 vs. 84 hours) and tolerance of a solid diet (60 vs. 72 hours) in those who received valdecoxib (Sim 1.2007). A systematic review of 34 randomized trials from January 2002 to January 2012 concluded that a multimodal analgesic regimen that utilized early feeding in conjunction with local analgesics, nonsteroidal anti-inflammatory medications, or spinal analgesia with limitation on morphine utilization was associated with significant acceleration of bowel motility. ${ }^{22}$ The aforementioned studies provide substantial evidence that a multimodal approach to pain control so as to limit need for opiate use hastens return of bowel function and reduces the incidence of POI.

\section{Epidural Use}

There have been several studies investigating the effect of epidural use on POI. A systematic review and meta-analysis evaluated the use of epidural analgesia compared with alternative analgesic techniques after open abdominal surgery in the setting of enhanced recovery. Seven randomized trials were included from 1996 to 2013, and the review showed a faster return of bowel function as demonstrated by time to passage of flatus or bowel movement with use of epidural analgesia, but no difference in the rate of POI or hospital length of stay. ${ }^{23}$ While no significant difference in complication rate was detected, subgroup analysis showed fewer complications in the PCA group compared with the epidural group (OR: 1.97; 95\% CI: $1.10-3.53 ; p=0.02$ ).

In laparoscopic colectomy, specifically, a case-matched retrospective review of the Nationwide Inpatient Sample in 191,576 patients from 2002 to 2010 demonstrated no difference in the rates of POI between those who received an epidural and those who did not, while hospital length of stay, charges, and urinary tract infection rates were increased in the epidural group. ${ }^{24}$ These results were corroborated in an analysis of more than 29,000 patients who underwent laparoscopic colectomy using the University HealthSystem Consortium database from 2008 to 2014 . This study demonstrated no significant difference in the rates of POI when those who received an epidural were compared with those who had conventional analgesia, while total hospital charges and length of stay were greater in patients who had an epidural. ${ }^{25}$ A metaanalysis of seven randomized, controlled trials comparing epidural anesthesia versus patient controlled anesthesia was performed in patients who underwent laparoscopic colorectal surgery, which again found no significant difference in the rates of POI; in contrast to the aforementioned studies, no difference in hospital length of stay or urinary tract infection was noted. ${ }^{26}$ Based on the earlier data, epidural anesthesia use in colorectal surgery does not seem to affect the development of POI, especially in the setting of laparoscopic and enhanced recovery surgery pathways.

\section{Transversus Abdominis Plane Block}

A transversus abdominis plane (TAP) block involves instillation of local anesthetic into the neurovascular plane between the internal oblique and transversus abdominis muscles to block the sensory nerves of the anterior abdominal wall. This method has been evaluated in the context of laparoscopic colorectal surgery. A prospective, nonrandomized, blinded study compared the effect of local wound infiltration with and without TAP block in 48 patients who underwent laparoscopic colorectal resection. The authors found a reduction in opiate use, nausea, time to flatus, and time to tolerance of an oral diet; however, no difference in the rate of POI development was observed. ${ }^{27}$ A prospective, randomized, doubleblinded trial compared laparoscopic TAP block versus placebo in 79 patients who underwent laparoscopic colorectal resection and found a reduction in pain scores but no difference in postoperative nausea or vomiting, opioid use, length of stay, or readmission; the rate of POI was not specifically analyzed. ${ }^{28} \mathrm{~A}$ Cochrane review of eight studies on the effect of TAP blocks compared with any other mode of analgesia after abdominal surgery demonstrated no impact on nausea or vomiting, although the rate of ileus was not specifically investigated. ${ }^{29}$ Little data are present on the effect of TAP blocks on development of POI in the colorectal literature, but postoperative nausea and vomiting does not seem to be significantly affected.

\section{Chewing Gum}

The beneficial effect of chewing gum on shortening the duration of postoperative ileus has been debated. A randomized trial of patients who underwent laparoscopic colorectal resection 
and followed an enhanced recovery program was performed in which 41 patients were given chewing gum three times daily starting from the day of admission through discharge, and compared with 41 patients who did not. They found that time to passage of flatus was shorter (18 vs. 34 hours; $p=0.007$ ), and first bowel movement occurred earlier (19 vs. 44 hours; $p=0.001$ ) in the group that was given chewing gum, although there was no difference in hospital length of stay. ${ }^{30}$ The systematic review of 34 randomized trials by Wallström and Frisman mentioned earlier also demonstrated inconclusive results of gum chewing on recovery of gastrointestinal motility. ${ }^{22}$ While strong evidence is lacking in support of chewing gum as a means to reduce POI following segmental colon resection, there is little downside to its use in patients who are so inclined.

\section{Treatment}

In an ideal world, POI could be prevented by risk factor modification. However, despite best efforts, a significant amount of patients who undergo elective colorectal surgery will develop POI. Treatment is supportive. Bowel rest is employed with use of a nasogastric tube when necessary, and institution of parenteral nutrition in prolonged cases. Enteral nutrition is reinstituted gradually as tolerated based on clinical judgment once bowel function has returned and distension is improved.

\section{Conclusion}

Postoperative ileus is temporary inhibition of gastrointestinal motility after surgical intervention due to nonmechanical causes that prevents sufficient oral intake. Specific and uniform criteria for diagnosis are still lacking. Its occurrence following elective colorectal surgery appears to be between 10 and $30 \%$ of patients, and it can lead to prolonged length of stay and increased postoperative morbidity. There is significant evidence to suggest that a minimally invasive approach and multimodal pain regimens reduce the development of POI, while the use of epidural analgesia does not have a significant effect. The beneficial effect of chewing gum, alvimopan, TAP blocks, and ERAS protocols may decrease development of POI in selected groups of patients who undergo elective colorectal surgery, and shorten time to return of bowel function, but overall the data remain inconclusive.

\section{Conflict of Interest \\ None declared.}

\section{Acknowledgment}

No financial support or personal assistance has been utilized for this work.

\section{References}

1 Wolthuis AM, Bislenghi G, Fieuws S, de Buck van Overstraeten A, Boeckxstaens G, D'Hoore A. Incidence of prolonged postoperative ileus after colorectal surgery: a systematic review and metaanalysis. Colorectal Dis 2016;18(01):01-09

2 Venara A, Neunlist M, Slim K, et al. Postoperative ileus: pathophysiology, incidence, and prevention. J Visc Surg 2016;153(06): 439-446

3 Gero D, Gié O, Hübner M, Demartines N, Hahnloser D. Postoperative ileus: in search of an international consensus on definition, diagnosis, and treatment. Langenbecks Arch Surg 2017;402(01):149-158

4 Artinyan A, Nunoo-Mensah JW, Balasubramaniam S, et al. Prolonged postoperative ileus-definition, risk factors, and predictors after surgery. World J Surg 2008;32(07):1495-1500

5 Bragg D, El-Sharkawy AM, Psaltis E, Maxwell-Armstrong CA, Lobo DN. Postoperative ileus: recent developments in pathophysiology and management. Clin Nutr 2015;34(03):367-376

6 Vather R, O'Grady G, Bissett IP, Dinning PG. Postoperative ileus: mechanisms and future directions for research. Clin Exp Pharmacol Physiol 2014;41(05):358-370

7 Vather R, Bissett IP. Risk factors for the development of prolonged post-operative ileus following elective colorectal surgery. Int $\mathrm{J}$ Colorectal Dis 2013;28(10):1385-1391

8 Grass F, Slieker J, Jurt J, et al. Postoperative ileus in an enhanced recovery pathway-a retrospective cohort study. Int J Colorectal Dis 2017;32(05):675-681

9 Vather R, Josephson R, Jaung R, Robertson J, Bissett I. Development of a risk stratification system for the occurrence of prolonged postoperative ileus after colorectal surgery: a prospective risk factor analysis. Surgery 2015;157(04):764-773

10 Schwenk W, Böhm B, Haase O, Junghans T, Müller JM. Laparoscopic versus conventional colorectal resection: a prospective randomised study of postoperative ileus and early postoperative feeding. Langenbecks Arch Surg 1998;383(01):49-55

11 Fujii S, Ishibe A, Ota M, et al. Short-term results of a randomized study between laparoscopic and open surgery in elderly colorectal cancer patients. Surg Endosc 2014;28(02):466-476

$12 \mathrm{Ng}$ WQ Neill J. Evidence for early oral feeding of patients after elective open colorectal surgery: a literature review. J Clin Nurs 2006;15(06):696-709

13 MacKay G, Ihedioha U, McConnachie A, Serpell M, Molloy RG, O'Dwyer PJ. Laparoscopic colonic resection in fast-track patients does not enhance short-term recovery after elective surgery. Colorectal Dis 2007;9(04):368-372

14 Carmichael JC, Keller DS, Baldini G, et al. Clinical practice guidelines for enhanced recovery after colon and rectal surgery from the American Society of Colon and Rectal Surgeons and Society of American Gastrointestinal and Endoscopic Surgeons. Dis Colon Rectum 2017;60(08):761-784

15 Xu LL, Zhou XQ, Yi PS, Zhang M, Li J, Xu MQ. Alvimopan combined with enhanced recovery strategy for managing postoperative ileus after open abdominal surgery: a systematic review and meta-analysis. J Surg Res 2016;203(01):211-221

16 Nair A. Alvimopan for post-operative ileus: what we should know? Acta Anaesthesiol Taiwan 2016;54(03):97-98

17 Adam MA, Lee LM, Kim J, et al. Alvimopan provides additional improvement in outcomes and cost savings in enhanced recovery colorectal surgery. Ann Surg 2016;264(01):141-146

18 Keller DS, Flores-Gonzalez JR, Ibarra S, Mahmood A, Haas EM. Is there value in alvimopan in minimally invasive colorectal surgery? Am J Surg 2016;212(05):851-856

19 Vather R, Josephson R, Jaung R, Kahokehr A, Sammour T, Bissett I. Gastrografin in prolonged postoperative ileus: a double-blinded randomized controlled trial. Ann Surg 2015;262(01):23-30

20 Chen JY, Ko TL, Wen YR, et al. Opioid-sparing effects of ketorolac and its correlation with the recovery of postoperative bowel function in colorectal surgery patients: a prospective randomized double-blinded study. Clin J Pain 2009;25(06): 485-489 
21 Sim R, Cheong DM, Wong KS, Lee BM, Liew QY. Prospective randomized, double-blind, placebo-controlled study of pre- and postoperative administration of a COX-2-specific inhibitor as opioid-sparing analgesia in major colorectal surgery. Colorectal Dis 2007;9(01):52-60

22 Wallström A, Frisman GH. Facilitating early recovery of bowel motility after colorectal surgery: a systematic review. J Clin Nurs 2014;23(1-2):24-44

23 Hughes MJ, Ventham NT, McNally S, Harrison E, Wigmore S. Analgesia after open abdominal surgery in the setting of enhanced recovery surgery: a systematic review and meta-analysis. JAMA Surg 2014;149(12):1224-1230

24 Halabi WJ, Kang CY, Nguyen VQ et al. Epidural analgesia in laparoscopic colorectal surgery: a nationwide analysis of use and outcomes. JAMA Surg 2014;149(02):130-136

25 daSilva M, Lomelin D, Tsui J, Klinginsmith M, Tadaki C, Langenfeld S. Pain control for laparoscopic colectomy: an analysis of the incidence and utility of epidural analgesia compared to conventional analgesia. Tech Coloproctol 2015;19(09):515-520
26 Liu $\mathrm{H}, \mathrm{Hu} \mathrm{X}$, Duan $\mathrm{X}$, Wu J. Thoracic epidural analgesia (TEA) vs. patient controlled analgesia (PCA) in laparoscopic colectomy: a meta-analysis. Hepatogastroenterology 2014;61 (133):1213-1219

27 Pedrazzani C, Menestrina N, Moro M, et al. Local wound infiltration plus transversus abdominis plane (TAP) block versus local wound infiltration in laparoscopic colorectal surgery and ERAS program. Surg Endosc 2016;30(11):5117-5125

28 Keller DS, Ermlich BO, Schiltz N, et al. The effect of transversus abdominis plane blocks on postoperative pain in laparoscopic colorectal surgery: a prospective, randomized, double-blind trial. Dis Colon Rectum 2014;57(11):1290-1297

29 Charlton S, Cyna AM, Middleton P, Griffiths JD. Perioperative transversus abdominis plane (TAP) blocks for analgesia after abdominal surgery. Cochrane Database Syst Rev 2010;(12): CD007705

30 Shum NF, Choi HK, Mak JC, Foo DC, Li WC, Law WL. Randomized clinical trial of chewing gum after laparoscopic colorectal resection. Br J Surg 2016;103(11):1447-1452 